\title{
Allele-Specific Isothermal Amplification Method Using Unmodified Self-Stabilizing Competitive Primers
}

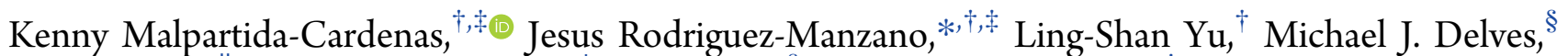 \\ Chea Nguon, "Kesinee Chotivanich, ${ }^{\perp}$ Jake Baum, ${ }^{\S}$ and Pantelis Georgiou ${ }^{\dagger}$
}

${ }^{\dagger}$ Centre for Bio-Inspired Technology, Department of Electrical and Electronic Engineering, Imperial College London, London, SW7 2AZ, United Kingdom

${ }^{\S}$ Department of Life Sciences, Imperial College London, South Kensington Campus, SW7 2AZ, London, United Kingdom

"National Centre for Parasitology, Entomology and Malaria Control, Phnom Penh 12302, Cambodia

${ }^{\perp}$ Department of Clinical Tropical Medicine, Faculty of Tropical Medicine, Mahidol University, Bangkok 10400, Thailand

\section{Supporting Information}

ABSTRACT: Rapid and specific detection of single nucleotide polymorphisms (SNPs) related to drug resistance in infectious diseases is crucial for accurate prognostics, therapeutics and disease management at point-of-care. Here, we present a novel amplification method and provide universal guidelines for the detection of SNPs at isothermal conditions. This method, called USS-sbLAMP, consists of SNP-based loop-mediated isothermal amplification (sbLAMP) primers and unmodified self-stabilizing (USS) competitive primers that robustly delay or prevent unspecific amplification. Both sets of primers are incorporated into the same reaction mixture, but always targeting different alleles; one set specific to the wild type allele and the other to the mutant allele. The mechanism of action relies on thermodynamically favored hybridization of totally complementary primers, enabling allele-specific amplification. We successfully validate our method by detecting SNPs, C580Y and Y493H, in the Plasmodium falciparum kelch 13 gene that are responsible for resistance to artemisinin-based combination therapies currently used globally in the treatment of malaria. USS-sbLAMP primers can efficiently discriminate between SNPs with high sensitivity (limit of detection of $5 \times 10^{1}$ copies per reaction), efficiency, specificity and rapidness (<35 min) with the capability of quantitative measurements for point-of-care diagnosis, treatment guidance, and epidemiological reporting of drug-resistance.
$\mathrm{T}$ he emergence of drug resistance is a constant threat to global public health, limiting the ability to treat infectious diseases effectively and compromising medical procedures. ${ }^{1}$ Rapid detection of single nucleotide polymorphisms (SNPs) that are linked with resistance phenotypes in infectious pathogens is, therefore, key to improving treatment efficacy and guiding clinical usage of drugs toward development of personalized medicine. However, the detection of genetic markers is still a major challenge for molecular-based diagnostic technologies, especially for those aiming to be deployed at point-of-care (PoC).

Currently, high-throughput methods such as Next Generation Sequencing or Sanger sequencing are considered the gold standard for low frequency allele detection. Despite their advancement in the past decade, their high cost and time to report results limit their use outside specialized laboratories. ${ }^{2,3}$ A variety of PCR-based methods have been developed to enhance detection of genetic markers. These allele-specific PCR (AS-PCR) techniques can be classified into two main groups: major allele suppression methods and minor allele enrichment methods. Within the first group, blocking primers have been designed to suppress the amplification of the major allele, enabling minor allele amplification by allele-specific primers. Different approaches have been reported such as (i) PCR blocking primers with a poly-A tail of four nucleotides ${ }^{4}$ or with 3 ' end modifications (ddNTPs, carbon-spacer, or inverted DNA nucleotides), ${ }^{5-7}$ (ii) PCR clamping ${ }^{6}$ based on the incorporation of peptide nucleic acids, ${ }^{8}$ bridged nucleic acids, ${ }^{9}$ or locked nucleic acids, ${ }^{10}$ or (iii) PCR amplification of previously treated DNA with lambda exonuclease. ${ }^{11}$ Within the second group, AS-PCR techniques have focused on enhancing the amplification of the minor allele ${ }^{12}$ by the incorporation of (i) primers specific to the SNP at their $3^{\prime}$ end, ${ }^{13}$ (ii) primers with mismatches within the four bases from the $3^{\prime}$ end, ${ }^{14,15}$ or (iii) primers of different lengths for melting temperature $\left(T_{\mathrm{m}}\right)$ analysis. ${ }^{16}$ However, the need of thermal cycling, gel electrophoresis, ${ }^{17}$ and modified oligonucleotides for validation

Received: May 30, 2018

Accepted: September 18, 2018

Published: September 18, 2018 
elevate the time and cost per sample, preventing these PCRbased methods from being widespread to decentralized areas.

An alternative to PCR is isothermal DNA amplification techniques, which offer nucleic acid synthesis at constant temperature using simpler and less complex equipment better suited to applications at PoC. Several isothermal techniques have been reported, each one with its own innovative characteristics and temperature requirements in the range of $30-65{ }^{\circ} \mathrm{C}$, depending on the enzymes used in the reaction. The most popular techniques are (i) recombinase polymerase amplification, ${ }^{18}$ (ii) nucleic acid sequence-based amplification, ${ }^{19}$ (iii) loop-mediated isothermal amplification (LAMP), ${ }^{20}$ and (iv) helicase-dependent amplification. ${ }^{21}$ Among them, LAMP has become popular due to advantages such as its high efficiency, high amplification yield, high specificity due to four to six primers in the reaction, strand displacement DNA synthesis between 60 and $65{ }^{\circ} \mathrm{C}$, and the capability of visual detection of products due to precipitation of magnesium pyrophosphate. $^{22,23}$ Allele-specific LAMP (AS-LAMP) has been previously described for minor allele amplification. Several approaches have been reported such as (i) placing the SNP at the $5^{\prime}$ end of overlapped FIP and BIP primers, ${ }^{24,25}$ (ii) placing the SNP at the $3^{\prime}$ end of B2 or LB primer, ${ }^{26}$ or (iii) introducing additional mismatches. ${ }^{27,28}$ These methods either delay the onset of the unspecific reaction, or the time-topositive (TTP) of the specific reaction is too late such that their applicability is limited to test high concentrated samples. Each design is unique and target dependent, preventing their standardization across other relevant SNPs. The addition of universal QProbe ${ }^{29}$ or the utilization of the Taq Mut enzyme ${ }^{30}$ have addressed this to an extent, however, the need of melting analysis, complex primer design, or low specificity prevent the use of these methods at the PoC.

Here, we describe a new method and propose universal primer design guidelines for enhanced SNP detection at isothermal conditions based on (i) SNP-based loop-mediated isothermal amplification (sbLAMP) primers, which consist of six primers targeting eight different regions, with two of them responsible for AS-LAMP amplification, and (ii) the novel unmodified self-stabilizing (USS) competitive primers, complementary to the SNP at their $5^{\prime}$ end, which are responsible for robustly delaying or preventing unspecific sbLAMP amplification. In contrast to conventional blocking PCR primers, which present $3^{\prime}$ end modifications, the proposed USS primers are chemically unmodified with capability to extend (higher stability) and compete with other primers and intermediate amplified structures in the reaction. We demonstrate successful application of our method, defining universal primer design guidelines based on the detection of the SNP C580Y located in the gene kelch 13 (K13) of the ATrich genome of $P$. falciparum responsible for resistance to artemisinin-based drug treatment of malaria. ${ }^{31,32}$ As validation, the same principles were used to develop USS-sbLAMP primers for detection of another antimalarial-resistant K13 SNP, Y493H. The proposed method has shown high sensitivity, efficiency, specificity and rapid TTP (<35 min) for SNP detection, and it is also applicable to mixed populations. The primer design guidelines we provide at the end of each section aim to give a foundation for the design of new USS-sbLAMP primer sets for the detection of other relevant targets. Diagnostic tests incorporating the proposed isothermal chemistry will greatly expand the capability of rapid SNP screening, including in limited-resource settings (LRS) where rapid, sensitive, and specific diagnostics of infectious diseases are of vital importance.

\section{EXPERIMENTAL SECTION}

Primer Design of USS-sbLAMP Specific to SNP C580Y and SNP Y493H. The USS-sbLAMP method consists of a total of eight primers targeting ten distinct regions of the DNA template. The sbLAMP primer set is composed of two outer primers (F3 and B3), two loop primers ( $\mathrm{LF}$ and $\mathrm{LB}$ ) and two inner primers (sbFIP and sbBIP), where $\mathrm{F} 1 \mathrm{c}$ and $\mathrm{B} 1 \mathrm{c}$ locate the SNP at their $5^{\prime}$ end. The USS primer set consists of a forward blocking competitive primer (FB) and a backward blocking competitive primer (BB). The USS-sbLAMP primer sets for the specific detection of C580Y and $Y 493 H$ were designed based on the gene K13 of P. falciparum. Consensus reference genomic sequences from all human-infective Plasmodium species (PF3D7 1343700.1, PFIT 1342900, PKNH 1257700, PKNOH S09541100, PVP01 1211100, PVX_083080, PmUG01_12021200, POcGH01_12019400) were retrieved from Plasmodium Genomic Resource (PlasmoDB $)^{33}$ and aligned using MUSCLE algorithm ${ }^{34}$ in Geneious 10.0.5 software. ${ }^{33}$ The sbLAMP primer set was designed using Primer Explorer V5 (Eiken Chemical Co. Ltd., Tokyo, Japan; http://primerexplorer.jp/lampv5e/index.html) and optimized manually to locate the SNP at the $5^{\prime}$ end of F1c and B1c, following the method described by Eiken Chemical Co., Ltd., ${ }^{24}$ with the novelty of considering the local GC\% composition for the design of FIP and BIP primers, named as sbFIP and sbBIP. Different lengths of $\mathrm{F} 1 \mathrm{c}$ and $\mathrm{B} 1 \mathrm{c}$ were designed for the SNP C580Y (17 bp, $19 \mathrm{bp}, 21 \mathrm{bp}$, and $25 \mathrm{bp}$ for F1c, and $21 \mathrm{bp}, 25$ bp, $27 \mathrm{bp}$, and $29 \mathrm{bp}$ for B1c) and for the SNP Y493H (21 bp for F1c and $17 \mathrm{bp}$ for B1c). The USS primer sets were designed based on the most suitable pair of F1c and B1c for allele-specific amplification based on experimental data (see Results and Discussion). FB and BB primers of different lengths were manually designed, always equal or longer than the selected F1c and B1c. They were between 0 and $6 \mathrm{bp}$ longer at the $3^{\prime}$ end (additional 0 to $-3.93 \mathrm{kcal} / \mathrm{mol}^{-1}$ ) and locate the SNP within the $3 \mathrm{bp}$ (up to $-1.31 \mathrm{kcal} / \mathrm{mol}^{-1}$ ) closer to their $5^{\prime}$ end (FB0, FB1, FB2 and BB0, BB1 and BB2), where the number indicates the position of the SNP from the $5^{\prime}$ end. To investigate the performance of the competitive primers, reverse-complementary primers to $\mathrm{FB} / \mathrm{BB}$, named FA/BA, were designed, and $3^{\prime}$ end modifications $(/ 3 \mathrm{AmMo} /)$ were added to each primer set, named $\mathrm{FA}^{*} / \mathrm{BA}^{*}$ and $\mathrm{FB}^{*} /$ $\mathrm{BB}^{*}$. All primers used in this paper can be found in Table S1 and final USS-sbLAMP primer sets in Table S2.

USS-sbLAMP Mechanism. The incorporation of USS primers in the sbLAMP reaction enhances allele discrimination by preventing unspecific sbLAMP amplification (caused by unspecific sbLAMP primers) through thermodynamically favored hybridization to the template due to total complementarity and self-stabilizing capability. USS primers and $\mathrm{sbFIP/sbBIP}$ primers (within sbLAMP) are in the same reaction mixture but always targeting different alleles, one set specific to the wild type (WT) allele and the other to the mutant (MT) allele. Allelic discrimination is possible by comparing the outcome of two independent reactions: WT reaction (sbLAMP ${ }_{\mathrm{WT}}$ and $\mathrm{USS}_{\mathrm{MT}}$ primers) and $\mathrm{MT}$ reaction (sbLAMP $_{\mathrm{MT}}$ and $\mathrm{USS}_{\mathrm{WT}}$ primers), as shown in Scheme 1. sbLAMP follows the LAMP method described by Notomi et $\mathrm{al}^{20}$ It is initiated by the binding of two inner primers sbFIP (F1c-F2) and sbBIP (B1c-B2), which bind to F2c and B2c 
Scheme 1. Mechanism of USS-sbLAMP in the Presence of Mutant DNA ${ }^{a}$
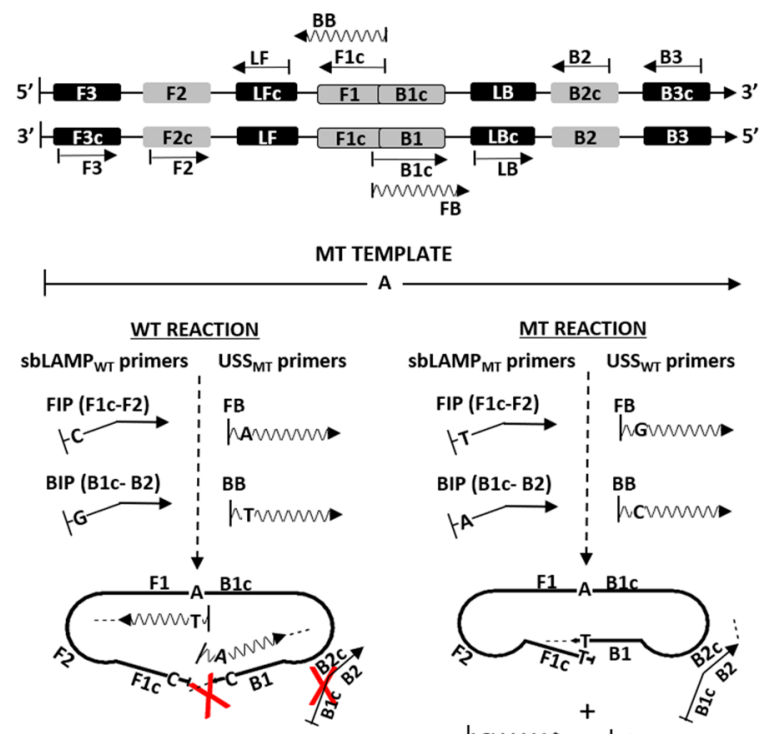

No Amplification

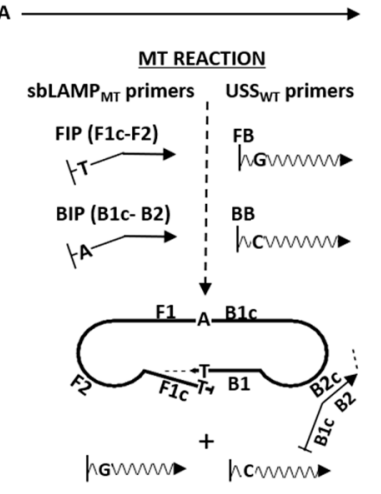

Amplification

${ }^{a}$ As an example, mutant (MT) DNA presents allele A and it is uniquely amplified within the MT reaction sbLAMP $_{\mathrm{MT}}$ and USS $_{\mathrm{WT}}$ primers). Amplification of the MT DNA within the wild type (WT) reaction (sbLAMP ${ }_{\mathrm{WT}}$ and $\mathrm{USS}_{\mathrm{MT}}$ primers) is significantly delayed or prevented by the annealing of complementary USS $_{\mathrm{MT}}$ primers to the template.

regions, respectively, leaving $\mathrm{F} 1 \mathrm{c}$ and $\mathrm{B} 1 \mathrm{c}$ free. Two outer primers $\mathrm{F} 3$ and $\mathrm{B} 3$ displace the strands, releasing singlestranded DNA (ssDNA). The free $\mathrm{F} 1 \mathrm{c}$ and $\mathrm{B} 1 \mathrm{c}$ form a dumbbell-like structure by annealing to their complementary sequences $\mathrm{F} 1$ and $\mathrm{B} 1$, respectively. It is rapidly linearized from its $3^{\prime}$ end, and the binding of sbFIP and sbBIP initiate the cyclic amplification step. During this step, LF and LB primers bind their complementary sequences, which are in between $\mathrm{B} 1 \mathrm{c}$ and $\mathrm{B} 2 \mathrm{c}$ and $\mathrm{F} 1$ and $\mathrm{F} 2$, further accelerating the reaction. ${ }^{36}$

The complementarity of the USS primers to the target template (with SNP specificity) suggests the possibility of hybridization during the initiation stage of sbLAMP, causing a general delay in specific and unspecific reactions until USS primers are displaced by F3/B3 and sbFIP/sbBIP (F2 and B2, specifically). At the end of the initiation stage, the annealing of the USS primers to the template and the formation of the dumbbell-like structure with the binding of sbFIP and sbBIP cannot occur simultaneously. Consequently, there will be a competition and the most energetically favorable reaction, the specific reaction, will occur at a first stage. In case that the DNA template is not specific to the sequence of the USS primers, they may anneal, but the $5^{\prime}$ end will be unbound leaving a toehold for loop formation and subsequent amplification by specific sbLAMP primers. If the DNA template is specific to the sequence of the USS primers, a second stage will take place. The association between the USS primers and the template is not transient and a highly stable primer-template complex is formed as nucleotides are incorporated by the DNA polymerase. FB will anneal to the $B 1$ region of the dumbbell-like structure preventing the annealing between $\mathrm{B} 1$ and $\mathrm{B} 1 \mathrm{c}$ regions of this structure (this $3^{\prime}$ end cannot act as a primer) and the annealing of B2 of the $\mathrm{BIP}$ primer to the $\mathrm{B} 2 \mathrm{c}$ region of the dumbbell-like structure;

$\mathrm{BB}$ will anneal to the F1 region of the dumbbell-like structure preventing the annealing between $\mathrm{F} 1$ and $\mathrm{F} 1 \mathrm{c}$ regions of this structure (this $3^{\prime}$ end cannot act as a primer) and the annealing of F2 of the FIP primer to the F2c region of the dumbbell-like structure. This self-stabilizing behavior will create byproducts that do not prime amplification and, therefore, will inhibit the formation of dumbbell-like structures preventing unspecific amplification.

The hybridization energies of specific $\mathrm{FB} / \mathrm{BB}$ must be favorable with respect to the energies of unspecific F1c/B1c, $\Delta G^{\circ}{ }_{(\mathrm{F} 1 \mathrm{c} / \mathrm{B} 1 \mathrm{c})}>\Delta G^{\circ}{ }_{(\mathrm{FB} / \mathrm{BB})}$. To achieve that, the design of USS primers followed two principles: primer elongation along the $3^{\prime}$ end and displacement of the position of the SNP from their $5^{\prime}$ end. Regarding the first principle, $\mathrm{FB} / \mathrm{BB}$ primers should be equal or longer than F1c/B1c. Overlapping with the $5^{\prime}$ end of LF and LB must be minimum (1-2 bp) and preferably avoided, although it might be tolerated since only one of the USS primers will be slightly compromised (some mismatches with the template, although it might be able to bind because the region is free) when preventing the formation of the dumbbell structure. Since USS primers should not delay the TTP, their free energy values should not exceed in more than $10 \mathrm{kcal} \mathrm{mol}^{-1}$ the free energy values of F1c/B1c. Regarding the second principle, placing the SNP slightly away from the $5^{\prime}$ end of USS primers might enhance their specificity due to the fact that a local region around the SNP will be opened or closed based on their complementarity at the SNP position. Depending on the sequence of the target, placing the SNP at their $5^{\prime}$ end could be the most optimum configuration.

Thermodynamic Calculations of USS-sbLAMP Primers. Possible secondary structures, primer dimer formation, and hybridization stability were checked using NUPACK ${ }^{37}$ and NetPrimer (Premier Biosoft, NetPrimer; https://www. premierbiosoft.com/netprimer/). Equations ${ }^{38-40}$ can be found in Table S3 and obtained $\Delta G^{\circ}$ values in Tables S4, S7, S9, and S11.

Samples and DNA Extraction Methods. Two gBlock Gene fragments of $607 \mathrm{bp}$ were purchased from Integrated DNA Technologies (Table S2) and resuspended in TE buffer to $5 \mathrm{ng} / \mu \mathrm{L}$ stock solutions (stored at $-20{ }^{\circ} \mathrm{C}$ ). The WT synthetic DNA template (named here as WT template) contained SNPs 580C and 493Y; the MT synthetic DNA template (named here as MT template) contained the corresponding drug-resistant mutations, $580 Y$ and $493 \mathrm{H}$. $P$. falciparum genomic DNA (gDNA) was isolated using the PureLink Genomic DNA Mini Kit (ThermoFisher Scientific) from Cambodian and Thai culture adapted asexual parasites harboring the WT K13 allele (ANL1 and ARN1G, respectively), a Thai isolate harboring K13 539T mutation (APS2G), a Cambodian isolate containing K13 493H mutation (ANL8G), and a Cambodian isolate with K13 580 Y mutation (ANL5G). P. ovale curtisi, $P$. ovale wallikeri, $P$. vivax, $P$. malariae, and P. knowlesi clinical isolates (gDNA) were kindly provided by Prof. Colin Sutherland. The samples were stored at $-20{ }^{\circ} \mathrm{C}$ until experiments were performed.

USS-sbLAMP Reaction Conditions. Two independent reactions, WT reaction and MT reaction, were performed with each target. Each reaction mixture contained the following: 1.5 $\mu \mathrm{L}$ of $10 \times$ isothermal buffer, $0.9 \mu \mathrm{L}$ of $\mathrm{MgSO}_{4}(100 \mathrm{mM}$ stock), $2.1 \mu \mathrm{L}$ of dNTPs ( $10 \mathrm{mM}$ stock), $0.375 \mu \mathrm{L}$ of BSA (20 $\mathrm{mg} / \mathrm{mL}$ stock), $2.4 \mu \mathrm{L}$ of Betaine ( $5 \mathrm{M}$ stock), $0.375 \mu \mathrm{L}$ of SYTO 9 Green (20 $\mu \mathrm{M}$ stock), $0.6 \mu \mathrm{L}$ of Bst 2.0 DNA polymerase $(8,000 \mathrm{U} / \mathrm{mL}$ stock $), 3 \mu \mathrm{L}$ of different 
A

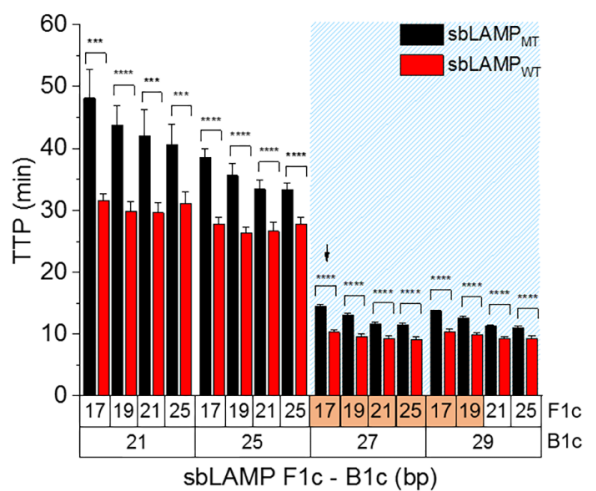

B

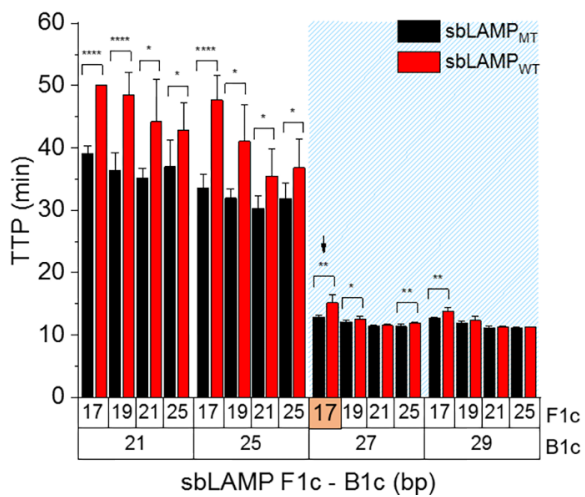

C

\begin{tabular}{|c|c|c|c|c|c|}
\hline Allele & $\begin{array}{l}\text { F1c } \\
\text { B1c }\end{array}$ & 17 bp & 19 bp & $21 \mathrm{bp}$ & $25 \mathrm{bp}$ \\
\hline \multirow{4}{*}{$\begin{array}{l}\text { WT } \\
580 \mathrm{C}\end{array}$} & $21 \mathrm{bp}$ & 16.5 & 14.0 & 12.4 & 9.6 \\
\hline & $25 \mathrm{bp}$ & 10.7 & 9.3 & 6.8 & 5.5 \\
\hline & $27 \mathrm{bp}$ & 4.2 & 3.4 & 2.4 & 2.3 \\
\hline & 29 bp & 3.3 & 2.8 & 1.9 & 1.7 \\
\hline \multirow{4}{*}{$\begin{array}{l}\text { MT } \\
580 \mathrm{Y}\end{array}$} & $21 \mathrm{bp}$ & 10.9 & 12.2 & 9.1 & 5.9 \\
\hline & $25 \mathrm{bp}$ & 14.2 & 9.1 & 5.1 & 5.0 \\
\hline & $27 \mathrm{bp}$ & 2.3 & 0.4 & 0.1 & 0.5 \\
\hline & $29 \mathrm{bp}$ & 1.1 & 0.4 & 0.1 & 0.1 \\
\hline
\end{tabular}

Figure 1. Results of sbLAMP using different F1c-B1c primer lengths. (A) Comparison of WT template (580C) amplification by WT specific $\left(\operatorname{sbLAMP}_{\mathrm{WT}}\right)$ and MT specific (sbLAMP ${ }_{\mathrm{MT}}$ ) primer sets with different lengths of F1c and B1c. (B) Comparison of MT template (580Y) amplification by WT specific (sbLAMP ${ }_{\mathrm{WT}}$ ) and MT specific $\left(\right.$ sbLAMP $_{\mathrm{MT}}$ ) primer sets with different lengths of F1c and B1c. (C) Table showing the $\Delta$ TTP values between specific and unspecific primer sets. Average TTP values of specific reactions below 20 min are blue shadowed. $\Delta$ TTP values above $2 \mathrm{~min}$ are orange colored. Average of two experiments performed in triplicates using $5 \times 10^{4}$ copies/reaction of synthetic DNA. Selected sbLAMP primer sets are indicated with arrows in the plots and dark blue cells in the table.
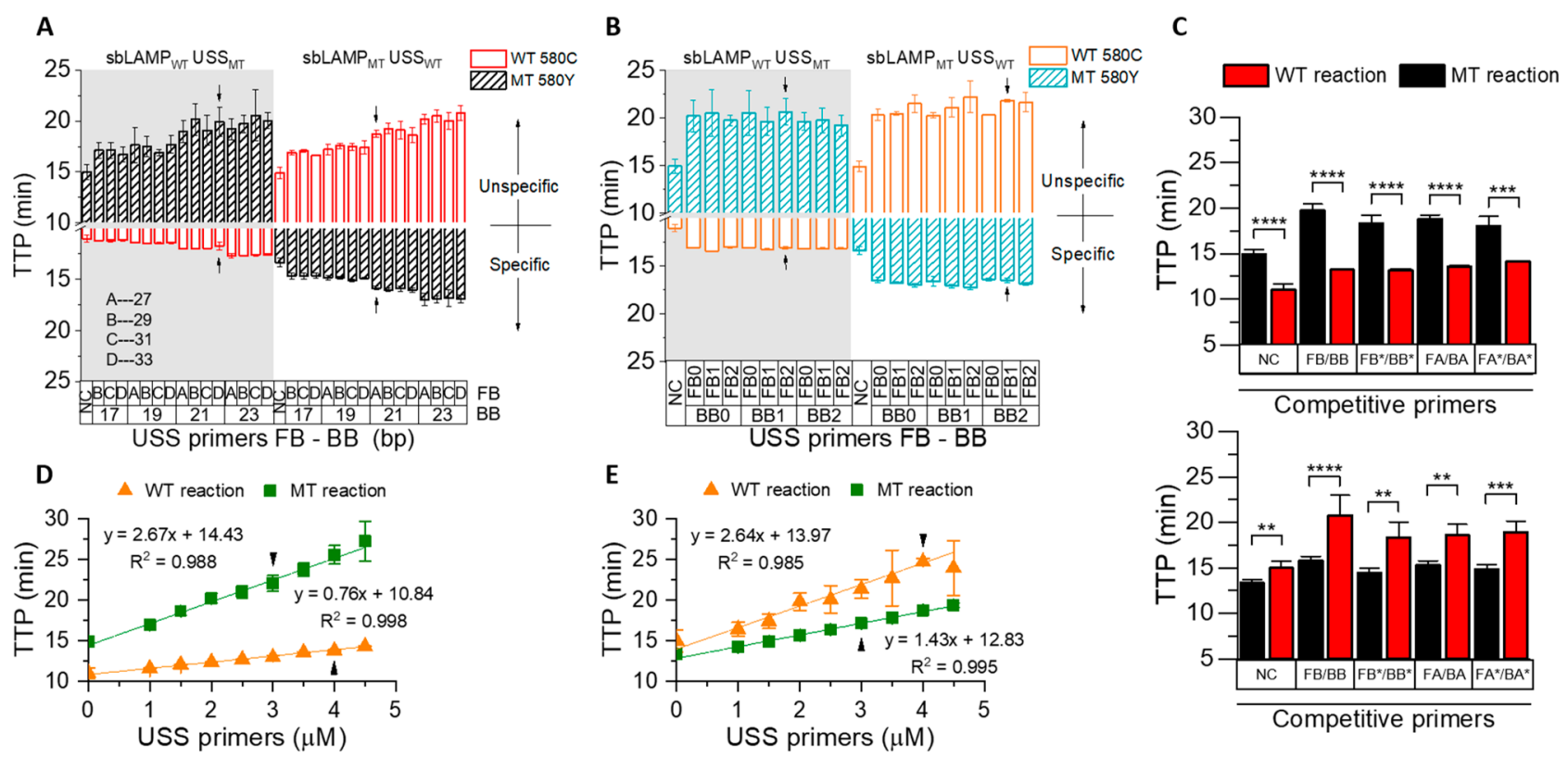

Figure 2. Incorporation of USS primer for enhancement of sbLAMP. (A) Performance comparison of USS primers with different lengths added to sbLAMP reaction at $2 \mu \mathrm{M} /$ reaction for detecting SNP C580Y. A total of 15 different combinations were tested. Selected USS primers are indicated with arrows (****p-value). (B) Performance comparison of redesigned USS primers added to sbLAMP reaction at $2 \mu \mathrm{M} /$ reaction for detecting SNP C580Y. A total of 9 different combinations were tested. Selected USS primers are indicated by arrows (****p-value). (C) Comparison of modified, unmodified and reverse competitive primers for allele-specific detection (WT template in the upper section, and MT template in the lower section). (D, E) Optimisation of the concentration of USS primers (FB/BB). WT template (580C) in (D) and MT template (580Y) in (E). Selected concentrations for the detection of SNP C580Y are labeled by arrows, USS $\mathrm{MT}_{\text {at }} 4 \mu \mathrm{M}$ and $\mathrm{USS}_{\mathrm{WT}}$ at $3 \mu \mathrm{M}$. Average of two experiments performed in triplicates using $5 \times 10^{4}$ copies/reaction of synthetic DNA. Negative control (NC), no addition of USS primers.

concentrations of synthetic DNA or gDNA, $1.5 \mu \mathrm{L}$ of $10 \times$ sbLAMP primer mixture $(20 \mu \mathrm{M}$ sbBIP/sbFIP, $10 \mu \mathrm{M} \mathrm{LF} / \mathrm{LB}$, and $2.5 \mu \mathrm{M} \mathrm{B} 3 / \mathrm{F} 3)$, and enough nuclease-free water (ThermoFisher Scientific) to bring the volume to $15 \mu \mathrm{L}$. All reagents were purchased to New England BioLabs and all synthetic DNA to Integrated DNA Technologies. Reactions were performed at $63{ }^{\circ} \mathrm{C}$ for $50 \mathrm{~min}$ for screening purposes and 30-35 min for final assays. For high resolution melting, SYTO 9 Green was replaced by EvaGreen (Biotium, California) at a final concentration of $0.6 \mu \mathrm{M}$ and one cycle was performed at $95^{\circ} \mathrm{C}$ for $60 \mathrm{~s}, 40{ }^{\circ} \mathrm{C}$ for $60 \mathrm{~s}, 65^{\circ} \mathrm{C}$ for $1 \mathrm{~s}$, and $97{ }^{\circ} \mathrm{C}$ for $1 \mathrm{~s}$ (Figure S1). Experiments were performed twice, and each condition was run in triplicates $(5 \mu \mathrm{L}$ each reaction) loading the reactions into LightCycler 480 Multiwell Plates 96 (Roche Diagnostics) utilizing a LightCycler 96 RealTime PCR System (Roche Diagnostics). Competitive primers (FA/BA, $\mathrm{FA}^{*} / \mathrm{BA}^{*}, \mathrm{FB} / \mathrm{BB}$, or $\mathrm{FB}^{*} / \mathrm{BB}^{*}$ ) were incorporated into the sbLAMP $10 \times$ primer mixture at different final concentrations: $1,1.5,2,2.5,3,3.5,4$, and $4.5 \mu \mathrm{M}$. Nucleasefree water was adjusted to bring the volume to $15 \mu \mathrm{L}$. sbLAMP and USS primers were purchased from Integrated DNA Technologies and resuspended in nuclease-free water to 100 and $400 \mu \mathrm{M}$ stock solutions, respectively. The solutions were stored at $4{ }^{\circ} \mathrm{C}$. 

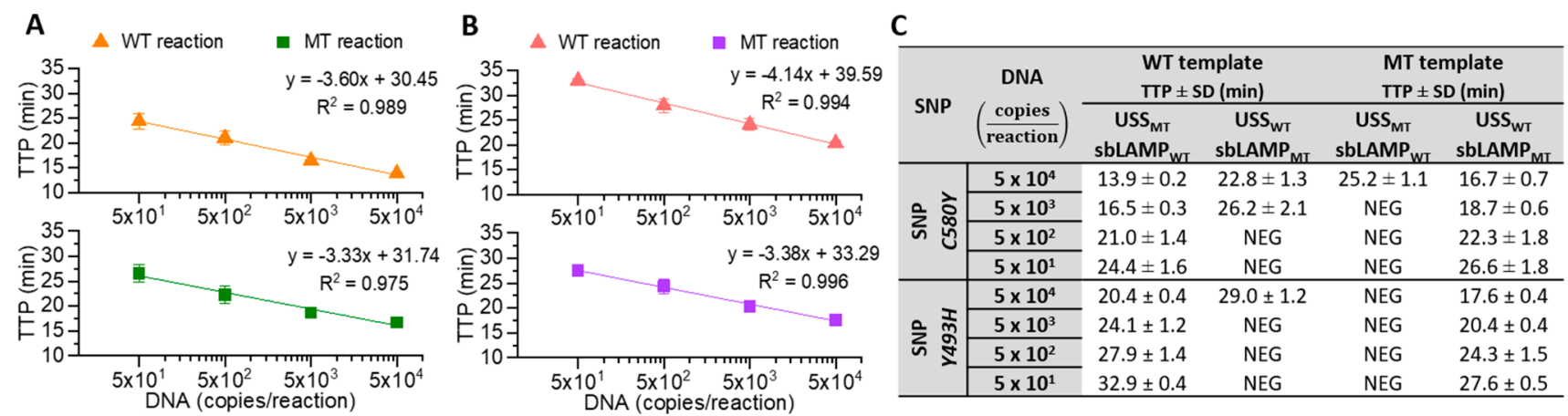

Figure 3. Standard curves of specific USS-sbLAMP reactions for detection of SNP C580Y and SNP Y493H. (A) USS-sbLAMP amplification of serially diluted WT template (580C) and MT template (580Y), in the upper and lower section, respectively. (B) USS-sbLAMP amplification of serially diluted WT template (493Y) and MT template (493H), in the upper and lower section, respectively. (C) Table showing the TTP values of WT and MT reactions with WT or MT template independently. Reactions were considered negative above 30 min for C580Y and above 35 min for Y493H. Average of two experiments performed in triplicates.

Sensitivity of USS-sbLAMP Method. Sensitivity was evaluated using 10-fold serial dilutions of synthetic DNA: $5 \times$ $10^{4}, 5 \times 10^{3}, 5 \times 10^{2}$, and $5 \times 10^{1}$ copies/reaction. Standard curves were generated by plotting the TTP against copies/ reaction with errors at one standard deviation. Sensitivity of mixed populations was evaluated by spiking synthetic DNA harboring the WT allele and the MT allele at different ratios $(100 / 0,80 / 20,50 / 50.20 / 80$, and $0 / 100$, in percentages) from stock solution at $2.5 \times 10^{3}$ copies/reaction.

Cross-Reactivity of USS-sbLAMP Method and Detection of Clinical Isolates. Ten clinical isolates (gDNA) were used to evaluate the feasibility of USS-sbLAMP and to prove the absence of cross-reactivity with any other human-infective Plasmodium species. Samples included P. ovale curtisi, $P$. ovale wallikeri, $P$. vivax, $P$. malariae, $P$. knowlesi (2 samples), $P$. falciparum harboring WT K13 alleles (2 samples), P. falciparum harboring the MT allele 580Y, 493H, and 539T allele (3 different samples). Experiments were performed as described above.

Statistical Analysis. Data is presented as mean TTP \pm standard deviation; $p$-values were calculated by Student's heteroscedastic t-test, with a two-sided distribution. Statistically significant difference was considered as $* p$-value $<0.05$, $* * p$-value $<0.01, * * * p$-value $<0.001, * * * * p$-value $<0.0001$; $\mathrm{k}$-means cluster analysis and ANOVA test were performed in Origin software (OriginLab, Northampton, MA).

\section{RESULTS AND DISCUSSION}

sbLAMP Method. Different lengths of F1c and B1c were designed in order to study the impact of local GC\% content, $T_{\mathrm{m}}$ and primer length in (i) allele-specificity and (ii) TTP. The design of overlapping primers for allele detection is not trivial, and the dissimilarity of the sequence in terms of ACTG composition upstream and downstream the position of the SNP should be taken into account. In general, primers are equal in length $(23-24 \mathrm{bp})^{24}$ and are considered specific and stable as long as they present optimal GC\% content and $T_{\mathrm{m}}$ to anneal to the DNA template. ${ }^{41}$ Due to the fact that the GC\% content and $T_{\mathrm{m}}$ are different at local regions with respect to the position of the SNP, allele-specific primers sbFIP and sbBIP might be of different lengths. F1c and B1c of four different lengths each (Table S1) were designed with hybridization energies considered between -10.00 and $-23.00 \mathrm{kcal} \mathrm{mol}^{-1}$ (Table S4), increasing proportionally to the length of the primers and the GC\%. Real-time amplification experiments of
Table 1. Study of the USS-sbLAMP Method in Mixed Populations Harbouring SNPs C580Y and $\mathrm{Y}^{4} 93 \mathrm{H}^{a}$

\begin{tabular}{ccc}
$\begin{array}{c}\text { spiked DNA } \\
\text { WT/MT (\%) }\end{array}$ & $\begin{array}{c}\text { WT reaction 580C } \\
\text { TTP } \pm \text { SD }(\mathrm{min})\end{array}$ & $\begin{array}{c}\text { MT reaction 580Y } \\
\text { TTP } \pm \text { SD }(\mathrm{min})\end{array}$ \\
\hline 100/0 & $15.6 \pm 0.6$ & NEG \\
$80 / 20$ & $15.9 \pm 0.7$ & $19.5 \pm 0.9$ \\
$50 / 50$ & $16.2 \pm 0.7$ & $19.1 \pm 0.2$ \\
20/80 & $16.7 \pm 0.9$ & $18.6 \pm 0.4$ \\
0/100 & NEG & $18.1 \pm 0.4$ \\
spiked DNA & WT reaction 493Y & MT reaction 493H \\
WT/MT (\%) & TTP \pm SD (min) & TTP \pm SD (min) \\
100/0 & $23.0 \pm 1.9$ & NEG \\
$80 / 20$ & $23.7 \pm 1.4$ & $22.8 \pm 1.3$ \\
50/50 & $24.4 \pm 1.9$ & $21.1 \pm 1.8$ \\
20/80 & $25.9 \pm 1.9$ & $20.4 \pm 1.8$ \\
0/100 & NEG & $20.0 \pm 1.5$
\end{tabular}

${ }^{a}$ Spiked mixed populations at different ratios $(100 / 0,80 / 20,50 / 50$, $20 / 80$, and $0 / 100$, in percentages) from stock at $2.5 \times 10^{3}$ copies/ reaction. Average of two experiments performed in triplicates.

WT (Figure 1A) and MT (Figure 1B) templates were performed independently. Two independent reactions, WT specific $\left(\operatorname{sbLAMP}_{\mathrm{WT}}\right)$ and MT specific $\left(\operatorname{sbLAMP}_{\mathrm{MT}}\right)$, were tested with each template. A total of 16 combinations of F1cB1c were performed; F1c of 17, 19, 21, and $25 \mathrm{bp}$ and B1c of $21,25,27$, and $29 \mathrm{bp}$. For both DNA templates, the TTP of specific and unspecific reactions was reduced as B1c was elongated from 21 to $29 \mathrm{bp}$, and allele-specific detection was enhanced as F1c was shortened from 25 to $17 \mathrm{bp}$.

The amplification time difference ( $\Delta$ TTP) between specific and unspecific reactions with each template is presented in Figure 1C. Detailed TTP values of all reactions are reported in Table S5. The results in Figure 1 showed the existence of a critical length for B1c (>27 bp) at which the TTP was significantly reduced (highlighted in blue). Two highly significant clusters were obtained by performing k-means cluster analysis and ANOVA test of sbLAMP results of WT and MT templates $\left(P>F=4.44 \times 10^{-15}\right.$ and $P>F=1.53 \times$ $10^{-14}$, respectively). The selected sbLAMP primer sets according to the highest $\Delta$ TTP were $\mathrm{F} 1 \mathrm{c}_{17}-\mathrm{B} 1 \mathrm{c}_{27}$ for both alleles (indicated by arrows in Figure $1 \mathrm{~A}, \mathrm{~B}$, and dark blue cells in Figure 1C).

Guideline 1. Allele-specificity and TTP were significantly enhanced by modifying the length of $\mathrm{F} 1 \mathrm{c}$ and $\mathrm{B} 1 \mathrm{c}$ with respect to the standard sizes. It is recommended to design allele- 
Table 2. Cross-Validation of the USS-sbLAMP Method for Detection of SNP C580Y and SNP Y493 ${ }^{a}$

\begin{tabular}{|c|c|c|c|c|c|}
\hline sample & $\begin{array}{l}\text { WT reaction } 580 \mathrm{C} \\
\mathrm{TTP} \pm \mathrm{SD}(\mathrm{min})\end{array}$ & $\begin{array}{l}\text { MT reaction } 580 Y \\
\mathrm{TTP} \pm \mathrm{SD}(\mathrm{min})\end{array}$ & $\begin{array}{l}\text { WT reaction } 493 Y \\
\text { TTP } \pm \mathrm{SD}(\mathrm{min})\end{array}$ & $\begin{array}{l}\text { MT reaction } 493 \mathrm{H} \\
\mathrm{TTP} \pm \mathrm{SD}(\mathrm{min})\end{array}$ & $\begin{array}{c}\text { Pan-P } \\
\text { TTP } \pm \text { SD (min) }\end{array}$ \\
\hline $580 C^{1}$ & $17.8 \pm 0.2$ & NEG & $26.3 \pm 1.4$ & NEG & $10.5 \pm 0.7$ \\
\hline $580 C^{2}$ & $16.7 \pm 0.1$ & NEG & $25.0 \pm 1.6$ & NEG & $9.2 \pm 0.4$ \\
\hline $580 Y$ & NEG & $18.5 \pm 0.6$ & $25.9 \pm 1.8$ & NEG & $8.1 \pm 0.1$ \\
\hline $493 \mathrm{H}$ & $13.8 \pm 0.3$ & $21.8 \pm 0.9$ & NEG & $18.5 \pm 0.2$ & $7.2 \pm 0.1$ \\
\hline $539 \mathrm{~T}$ & $16.8 \pm 0.4$ & NEG & $26.1 \pm 1.8$ & NEG & $8.9 \pm 0.2$ \\
\hline Poc & NEG & NEG & NEG & NEG & $9.2 \pm 0.7$ \\
\hline Pow & NEG & NEG & NEG & NEG & $20.3 \pm 9.7$ \\
\hline $\mathrm{Pv}$ & NEG & NEG & NEG & NEG & $9.3 \pm 0.4$ \\
\hline Pm & NEG & NEG & NEG & NEG & $9.5 \pm 0.7$ \\
\hline $\mathrm{Pk}^{1}$ & NEG & NEG & NEG & NEG & $5.5 \pm 0.1$ \\
\hline $\mathrm{Pk}^{2}$ & NEG & NEG & NEG & NEG & $6.3 \pm 0.3$ \\
\hline NTC & NEG & NEG & NEG & NEG & NEG \\
\hline
\end{tabular}

${ }^{a}$ No cross-reactivity with any human-infective Plasmodium. Published Pan-Plasmodium primer set ${ }^{50}$ was used as positive control. Samples tested were: $580 C^{1}$ (P. falciparum WT K13 sample 1), $580 C^{2}$ (P. falciparum WT K13 sample 2), $580 Y$ (P. falciparum K13 580Y), 493 H (P. falciparum K13

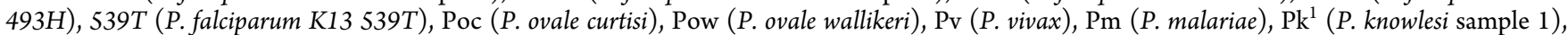
$\mathrm{Pk}^{2}$ (P. knowlesi sample 2), and NTC (non-template control). Experiment was performed in triplicates.

specific primers of different lengths depending on the GC\% content at the local region. Shortening the primer that sits at the richer GC\% region proved to enhance allele-specificity (preferably not less than $13 \mathrm{bp}$, i.e. $-8.52 \mathrm{kcal} \mathrm{mol}^{-1}$ ). Elongating the primer that sits at the richer AT\% region with respect to the other primer equalized their free energy values and $T_{\mathrm{m}}$ (even favoring the primer that sits at the richer AT\% region) such that both primers performed properly (early TTP). For optimal performance (allele-specificity and early TTP) more than $15 \mathrm{bp}$ difference $\left(-9.83 \mathrm{kcal} \mathrm{mol}^{-1}\right)$ between F1c and B1c should be avoided.

Incorporation of USS Primers for Enhancement of sbLAMP. USS primers were incorporated into the sbLAMP reaction at $2 \mu \mathrm{M}$ to prevent unspecific sbLAMP amplification. $\mathrm{FB}$ and $\mathrm{BB}$ of different lengths (FB of 27, 29, 31, and $33 \mathrm{bp}$ and $\mathrm{BB}$ of $17,19,21$, and $23 \mathrm{bp}$ ) were designed (Table S1) and a total of 15 different combinations were tested. Real-time amplification experiments of WT and MT templates were performed independently. Two independent reactions, WT reaction $\left(\operatorname{sbLAMP}_{\mathrm{WT}}\right.$ and $\mathrm{USS}_{\mathrm{MT}}$ ), and $\mathrm{MT}$ reaction $\left(\operatorname{sbLAMP}_{\mathrm{MT}}\right.$ and $\left.\mathrm{USS}_{\mathrm{WT}}\right)$, were tested with each template. The TTP of specific reactions are shown in the lower section of Figure $2 \mathrm{~A}$ and the TTP of unspecific reactions in the upper section. Detailed TTP values can be found in Table S6. As the length of USS primers is increased unspecific reactions are significantly delayed, while specific reactions are slightly delayed. Consequently, there is a trade-off between allelespecificity and sensitivity. The length of $\mathrm{BB}$ had a higher impact on the delay of both reactions than the length of FB. This behavior might be attributed to the richer GC\% content of the $\mathrm{BB}$ region and hence, the free energy hybridization values (Table S7). In general, USS primers must be within an optimal length range, and results of both templates, WT and MT, must be interpreted as a whole to select the most suitable USS primer set. The criterion for the selection of the most optimal size of $\mathrm{FB} / \mathrm{BB}$ was based on (i) minimizing the TTP of the specific reactions and (ii) maximizing the TTP of the unspecific reactions. Because both criteria cannot be fulfilled by any USS primer set, a balance is considered as the optimal. The selected lengths were $\mathrm{USS}_{\mathrm{WT}} \mathrm{FB}_{27} / \mathrm{BB}_{21}$ and $\mathrm{USS}_{\mathrm{MT}} \mathrm{FB}_{33} /$ $\mathrm{BB}_{21}$. The $\triangle \mathrm{TTP}$ between specific and unspecific reactions was statistically significant $(* * * * p$-value $)$ for both templates, WT and MT.

To enhance allele-specificity, the selected $\mathrm{FB} / \mathrm{BB}$ primers were redesigned to locate the SNP at three different positions from their $5^{\prime}$ end $\left(5^{\prime} \mathrm{X} \cdots 3^{\prime}\right.$ named as $\mathrm{FB} 0 / \mathrm{BB} 0,5^{\prime} \mathrm{NX} \cdots 3^{\prime}$ named as $\mathrm{FB} 1 / \mathrm{BB} 1$, and $5^{\prime} \mathrm{NNX} \cdots 3^{\prime}$ named as $\mathrm{FB} 2 / \mathrm{BB} 2$ where $\mathrm{X}$ denoted the SNP) by adding nucleotides totally complementary to the DNA template (Table S1). A total of nine different combinations were tested. Detailed TTP values can be found in Table S8. According to the estimated free energy values presented in Table S9, as far as the SNP is displaced away from the $5^{\prime}$ end, the specific hybridization is favored due to enhanced primer stability (additional -0.66 to $-1.31 \mathrm{kcal} \mathrm{mol}^{-1}$ ). However, the risk of creating an internal bulge between unspecific USS primers and the DNA template prevents to locate the SNP far from the third position (i.e., FB2/BB2) from the $5^{\prime}$ end of $\mathrm{FB} / \mathrm{BB}$ primers. TTP of specific reactions were not significantly affected by the incorporation of the redesigned USS primers. However, unspecific reactions were further delayed, contributing to allele-specific detection. Based on the experimental results obtained in Figure $2 \mathrm{~B}$ the selected redesigned primers were $\mathrm{USS}_{\mathrm{WT}} \mathrm{FB1}_{27} / \mathrm{BB}_{21}$ and $\mathrm{USS}_{\mathrm{MT}} \mathrm{FB} 2_{33} / \mathrm{BB}_{21}$. The $\Delta \mathrm{TTP}$ between specific and unspecific reactions was statistically significant (****p-value) for both templates, WT and MT.

Guideline 2. The nucleotide sequence of $\mathrm{FB} / \mathrm{BB}$ primers is restricted by the template, and their length is limited by the $5^{\prime}$ end of LF and LB primers. It is recommended that USS primers are between 0 to $8 \mathrm{bp}$ longer than F1c/B1c primers (additional 0 to $-5.24 \mathrm{kcal} \mathrm{mol}^{-1}$ ) with GC clamps at their $3^{\prime}$ end, if possible, to favor their stability upon hybridization. Locating the SNP within the three base pairs closer to their $5^{\prime}$ end (preferably FB1/BB1 or FB2/BB2, meaning an additional -0.66 to $-1.31 \mathrm{kcal} \mathrm{mol}^{-1}$ ) favor specific hybridization to their target template. Placing the SNP more than $4 \mathrm{bp}$ (additional $-2.62 \mathrm{kcal} \mathrm{mol}^{-1}$ ) away the $5^{\prime}$ end should be avoided due to the high risk of internal bulges formation and unspecific amplification.

Comparison of Modified, Unmodified, and Reverse Competitive Primers. Based on the selected $\mathrm{USS}_{\mathrm{WT}} \mathrm{FB1}_{27} /$ $\mathrm{BB}_{21}$ and $\mathrm{USS}_{\mathrm{MT}} \mathrm{FB} 2_{33} / \mathrm{BB}_{21}$ primers, their reverse-complement and the addition of $3^{\prime}$ end modifications were studied. 
Reverse-complement primers to $\mathrm{FB} / \mathrm{BB}$, named $\mathrm{FA} / \mathrm{BA}$, located the SNP at the $3^{\prime}$ end in contrast to $\mathrm{FB} / \mathrm{BB}$ which located the SNP at the $5^{\prime}$ end. The $3^{\prime}$ end modified versions of $\mathrm{FB} / \mathrm{BB}$ and $\mathrm{FA} / \mathrm{BA}$, named $\mathrm{FB}^{*} / \mathrm{BB}^{*}$ and $\mathrm{FA}^{*} / \mathrm{BA}^{*}$, included an amino modification which prevented the elongation of the primers by the DNA polymerase. The results presented in Figure 2C showed the similar behavior of the four primers in combination with sbLAMP. This data proved for the first time that unmodified primers $(\mathrm{FB} / \mathrm{BB}$ and $\mathrm{FA} / \mathrm{BA})$ can perform similarly or better for allele-specific detection than modified primers, based on statistical analysis. $t$ test was performed to compare the $\triangle \mathrm{TTP}$ values obtained using modified and unmodified primers with each template. In the case of the WT template, $\mathrm{FB} / \mathrm{BB}$ and $\mathrm{FA} / \mathrm{BA}$ performed better than their modified versions ( $p$-value $* * * *$ for both of them). In the case of the MT template, $\mathrm{FB} / \mathrm{BB}$ outperformed $\mathrm{FB}^{*} / \mathrm{BB}^{*}$ ( $p$-value $*)$, but no statistical significance was observed for FA/BA and $\mathrm{FA}^{*} / \mathrm{BA}^{*}$. Regarding the two sets of unmodified primers, FB/ $\mathrm{BB}$ presented higher $\triangle \mathrm{TTP}$ values than $\mathrm{FA} / \mathrm{BA}$. Compared to other strategies based on $3^{\prime}$ end chemical modifications, ${ }^{14,15,42}$ and the addition of mismatches at $3^{\prime}$ or $5^{\prime}$ end of FIP $^{28}$ or $\mathrm{BIP},{ }^{27}$ our methodology relies on total complementary unmodified primers with extension capability. The easier design and the lack of chemical modifications reduce the cost of the assay and production time, positioning this method as a promising molecular-based technique for SNP discrimination to be used at PoC.

Guideline 3. Unmodified primers performed similarly or better than $3^{\prime}$ end chemically modified primers to prevent unspecific amplification by sbLAMP, based on statistical analysis. The use of $\mathrm{FB} / \mathrm{BB}$ (SNP placed closed to the $5^{\prime}$ end) is recommended due to higher $\Delta \mathrm{TTP}$ between specific and unspecific reactions, easier design and lower cost and production time.

Study of Different Concentrations of USS Primers. The selected $\mathrm{USS}_{\mathrm{WT}} \mathrm{FB}_{27} / \mathrm{BB}_{21}$ and $\mathrm{USS}_{\mathrm{MT}} \mathrm{FB}_{23} / \mathrm{BB}_{21}$ primers were added to the sbLAMP reaction mix at different concentrations: $1,1.5,2,2.5,3,3.5,4$, and $4.5 \mu \mathrm{M}$. Real-time amplification experiments of WT and MT templates were performed independently. For each template, WT and MT reactions were tested (Figure 2D,E). Specific reactions were slightly delayed as the concentration of the USS primers was increased. The TTP values followed a linear fit with slopes of 0.76 and 1.48 for the WT and the MT template, respectively. Although the unspecific reactions also followed a linear fit, their slopes were sharper, denoting the significance of the concentration of $\mathrm{FB} / \mathrm{BB}$ on delaying unspecific amplification. The TTP of specific and unspecific reactions was evaluated to discern the optimum concentration of USS primers for allele discrimination. Analyzing all the combinations of different concentrations, the one presenting the highest $\Delta \mathrm{TTP}$ for both templates without compromising the other was selected as the optimum (Table S10). Selected concentrations were USS $_{\mathrm{WT}}$ at $3 \mu \mathrm{M}$ and $\mathrm{USS}_{\mathrm{MT}}$ at $4 \mu \mathrm{M}$.

Guideline 4. The concentration of USS primers in the reaction mixture should be higher (preferably between $1.5 \times$ to $2.5 \times)$ than the concentrations of sbFIP and sbBIP, to not compromise the limit of the detection of sbLAMP.

Sensitivity of USS-sbLAMP Method. Sensitivity was tested using 10 -fold serial dilutions $\left(5 \times 10^{4}, 5 \times 10^{3}, 5 \times 10^{2}\right.$, and $5 \times 10^{1}$ copies/reaction) of WT and MT templates independently (Figure 3A,C) for detection of SNP C580Y. Amplification curves are presented in Figure S2. For both alleles, the detection limit was $5 \times 10^{1}$ copies/reaction within $30 \mathrm{~min}$. Standard curves were generated with $R^{2}$ values of 0.989 and 0.975 , for WT and MT template, respectively, denoting the ability of the assay to robustly quantify samples. Pure WT and MT templates at concentrations below $5 \times 10^{3}$ and $5 \times 10^{4}$ copies/reaction, respectively, were uniquely amplified by their corresponding specific reactions providing "yes/no" results. Sensitivity of sbLAMP was not disrupted by the incorporation of the USS primers, and amplification of the nontarget template was successfully delayed or inhibited. Two reactions are always assessed (WT and MT reactions) for each sample, providing two TTP values being one of them negative at certain sample concentration (samples can be diluted if needed). Consequently, having always two reactions ensures the high specificity of the assay. Amplification was performed in less than $30 \mathrm{~min}$ at low DNA copies/reaction indicating the rapidness achieved utilizing the USS-sbLAMP method compared to other techniques based on PCR thermal cycling such as molecular beacons, TaqMan or FRET, ${ }^{43,44}$ which require complex designs, more than $1 \mathrm{~h}$ to finish and usually postamplification analysis. Other reported isothermal assays usually need between 20 to 75 min to finish, ${ }^{45,46}$ do not report quantitative data, ${ }^{47}$ rely on the addition of probes (Au NPs) to enhance specificity, ${ }^{48}$ lack amplification data at low concentrations, ${ }^{26}$ or require sequencing for product specificity. ${ }^{49}$

Validation of the USS-sbLAMP Method with the Artemisinin-Resistant SNP Y493H. Following the above created guidelines for the design of USS-sbLAMP primers, a specific USS-sbLAMP primer set was developed to detect a second K13 SNP, Y439H (Table S2). USS MT-sbLAMP $_{\text {WT }}$ primer set consisted of $\mathrm{F}_{1} \mathrm{c}_{21}-\mathrm{B} 1 \mathrm{c}_{17}+\mathrm{FB}_{24} / \mathrm{BB}_{26}$ at 4.5 $\mu \mathrm{M}$ and $\mathrm{USS}_{\mathrm{WT}^{-}} \mathrm{sbLAMP}_{\mathrm{MT}}$ primer set consisted of $\mathrm{F} 1 \mathrm{c}_{21}{ }^{-}$ $\mathrm{B} 1 \mathrm{c}_{17}+\mathrm{FB}_{24} / \mathrm{BBO}_{26}$ at $4.5 \mu \mathrm{M}$. Primer properties and hybridization energies are provided in Table S11. Sensitivity was tested using 10 -fold serial dilutions $\left(5 \times 10^{4}, 5 \times 10^{3}, 5 \times\right.$ $10^{2}$, and $5 \times 10^{1}$ copies/reaction) of WT and MT templates independently (Figure 3B,C). Amplification curves are presented in Figure S3. For both alleles, the limit of detection was $5 \times 10^{1}$ copies/reaction within $35 \mathrm{~min}$. Standard curves were generated with $R^{2}$ values of 0.994 and 0.996 , for WT and MT template. Pure WT and MT templates at concentrations below $5 \times 10^{4}$ copies/reaction and $5 \times 10^{5}$ copies $/$ reaction, respectively, were uniquely amplified by their corresponding specific reactions.

Study of the USS-sbLAMP Method in Mixed Populations Harboring SNPs C580Y and Y493H. USSsbLAMP primer sets for allele-specific detection of SNP C580Y and SNP $Y 493 H$ were evaluated with spiked mixed populations at different ratios $(100 / 0,80 / 20,50 / 50,20 / 80$, and $0 / 100$, in percentages). The four alleles were clearly discriminated within their respective specific reactions, as shown in Table 1. Unspecific reactions did not amplify, demonstrating the specificity of the method considering mixed populations at $80 / 20 \%$ as the limit for this experiment.

Cross-Validation of USS-sbLAMP Method for Detection of SNP C580Y and SNPY493H Using Clinical Isolates. The specificity of USS-sbLAMP for detecting SNP C580Y and $Y 493 \mathrm{H}$ was tested using gDNA samples from all human-infective Plasmodium species: P. falciparum, $P$. ovale curtisi, $P$. ovale wallikeri, $P$. vivax, $P$. malariae, and $P$. knowlesi, to prove the absence of cross-reactivity with any of them. In addition, three different $P$. falciparum samples harboring the mutations $580 Y, 493 \mathrm{H}$, and $539 \mathrm{~T}$ were tested. There was no 
cross-reactivity with any of the human-infective Plasmodium species (Table 2). The sample harboring the 539R mutation was uniquely amplified by the WT reactions 580C and 493Y, denoting the high specificity achieved by the MT reactions $580 Y$ and $493 \mathrm{H}$, which only amplified the samples harboring their respective mutations.

\section{CONCLUSION}

The proposed USS-sbLAMP method achieves high sensitivity, efficiency, specificity, and rapidness (TTP $<35 \mathrm{~min}$ ) for the detection of SNPs at isothermal conditions suitable for PoC applications. For the first time here, judiciously designed sbLAMP primers for allele-specific amplification are combined with novel unmodified self-stabilizing (USS) competitive primers specific to the SNP at their $5^{\prime}$ end which robustly delay or prevent unspecific sbLAMP amplification. The special design, based on the local GC\% content at the SNP position enhanced allele specificity, and the superior concentration of USS primers suppressed significantly unspecific amplification offering an excellent linear working range with a limit of detection of $5 \times 10^{1}$ copies/reaction for the detection of two of the most important artemisinin-resistant SNPs C580Y and Y493H within 30 and $35 \mathrm{~min}$, respectively. This universal isothermal method uses chemically unmodified primers totally complementary to the target template except at the SNP position (either wild-type or mutant allele), which significantly reduces the cost of reagents and equipment avoiding the need of thermal cycling and electrophoresis for product validation. The guidelines we provide aim to enable others to develop their own USS-sbLAMP primer sets to detect any kind of SNP, which can relate to drug resistance, disease susceptibility or cancer development. Diagnostic platforms that use CMOSbased ISFET electrochemical biosensors, ${ }^{51,52}$ which identify targets using $\mathrm{pH}$-based nucleic acid amplification, will perfectly couple with the USS-sbLAMP method described here, with efforts currently underway to integrate these. Any diagnostic device that can incorporate the proposed method will greatly expand the capability of rapid SNP screening at PoC, including LRS where infectious disease diagnosis and rapid drug resistance screening are urgently needed.

\section{ASSOCIATED CONTENT}

\section{S Supporting Information}

The Supporting Information is available free of charge on the ACS Publications website at DOI: 10.1021/acs.analchem.8b02416.

Primer sequences, detailed TTP values, thermodynamic equations and values, high resolution melting, and standard amplification curves (PDF)

\section{AUTHOR INFORMATION}

\section{Corresponding Author}

*Phone: (+44) 02075940843. E-mail: j.rodriguez-manzano@ imperial.ac.uk.

\section{ORCID $\odot$}

Kenny Malpartida-Cardenas: 0000-0002-3874-8810

\section{Author Contributions}

The manuscript was written through contributions of all authors. All authors have given approval to the final version of the manuscript.

\section{Author Contributions}

†These authors contributed equally.

\section{Notes}

The authors declare no competing financial interest.

\section{ACKNOWLEDGMENTS}

This work was supported by EPSRC HiPEDS CDT [EP/ L016796/1 to K.M.-C.]; Medical Research Council (MRC) together with the National Science Technology Development Agency (NSTDA) of Thailand (Newton Fund award to [MR/ N012275/1 to J.B. and M.J.D.]); Wellcome Investigator Award [100993/Z/13/Z to J.B.]; and EPSRC Global Challenge Research Fund [EP/P510798/1 to P.G., J.B., and J.R.-M.]. We are grateful to everyone who participated in the TRAC clinical studies, funded UK Government Department for International Development (DFID). We wish to acknowledge Prof. Arjen Dondorp from the Mahidol-Oxford Research Unit in Bangkok for sample provision and Prof. Colin Sutherland from London School of Hygiene and Tropical Medicine.

\section{REFERENCES}

(1) World Health Organization (WHO). Antimicrobial Resistance, www.who.int/mediacentre/factsheets/fs194/en/ (accessed Feb 6, 2018).

(2) Shao, D.; Lin, Y.; Liu, J.; Wan, L.; Liu, Z.; Cheng, S.; Fei, L.; Deng, R.; Wang, J.; Chen, X.; et al. Sci. Rep. 2016, 6 (1), 22338.

(3) Highsmith, W. E. Molecular Diagnostics; Humana Press: Totowa, NJ, 2006; pp 85-109.

(4) Wu, L. R.; Chen, S. X.; Wu, Y.; Patel, A. A.; Zhang, D. Y. Nat. Biomed. Eng. 2017, 1 (9), 714-723.

(5) Dobosy, J. R.; Rose, S. D.; Beltz, K. R.; Rupp, S. M.; Powers, K. M.; Behlke, M. A.; Walder, J. A. BMC Biotechnol. 2011, 11 (1), 8097.

(6) Vestheim, H.; Jarman, S. N. Front. Zool. 2008, 5 (1), 12.

(7) Wang, H.; Jiang, J.; Mostert, B.; Sieuwerts, A.; Martens, J. W. M.; Sleijfer, S.; Foekens, J. A.; Wang, Y. J. Mol. Diagn. 2013, 15 (1), 6269.

(8) Chiou, C.-C.; Luo, J.-D.; Chen, T.-L. Nat. Protoc. 2007, 1 (6), 2604-2612.

(9) Sekiguchi, M.; Obika, S.; Somjing, R.; Imanishi, T. Nucleosides, Nucleotides Nucleic Acids 2005, 24 (5-7), 1097-1100.

(10) Morandi, L.; de Biase, D.; Visani, M.; Cesari, V.; De Maglio, G.; Pizzolitto, S.; Pession, A.; Tallini, G. PLoS One 2012, 7 (4), e36084.

(11) Wu, T.; Chen, W.; Yang, Z.; Tan, H.; Wang, J.; Xiao, X.; Li, M.; Zhao, M. Nucleic Acids Res. 2018, 46 (4), e24-e24.

(12) Ugozzoli, L.; Wallace, R. Methods 1991, 2 (1), 42-48.

(13) Wang, J.; Chuang, K.; Ahluwalia, M.; Patel, S.; Umblas, N.; Mirel, D.; Higuchi, R.; Germer, S. BioTechniques 2005, 39 (6), 885893.

(14) Stadhouders, R.; Pas, S. D.; Anber, J.; Voermans, J.; Mes, T. H. M.; Schutten, M. J. Mol. Diagn. 2010, 12 (1), 109-117.

(15) Ayyadevara, S.; Thaden, J. J.; Shmookler Reis, R. J. Anal. Biochem. 2000, 284 (1), 11-18.

(16) Chen, C.-H. PLoS One 2016, 11 (4), e0146865.

(17) Knapp, L. A. Single Nucleotide Polymorphisms. Methods in Molecular BiologyTM (Methods and Protocols); Humana Press: Totowa, NJ, 2009; pp 137-151.

(18) Piepenburg, O.; Williams, C. H.; Stemple, D. L.; Armes, N. A. PLoS Biol. 2006, 4 (7), e204.

(19) Compton, J. Nature 1991, 350 (6313), 91-92.

(20) Notomi, T.; Okayama, H.; Masubuchi, H.; Yonekawa, T.; Watanabe, K.; Amino, N.; Hase, T. Nucleic Acids Res. 2000, 28 (12), $63 \mathrm{e}$

(21) Vincent, M.; Xu, Y.; Kong, H. EMBO Rep. 2004, 5 (8), 795800.

(22) Niemz, A.; Ferguson, T. M.; Boyle, D. S. Trends Biotechnol. 2011, 29 (5), 240-250. 
(23) Poon, L. L. M. Clin. Chem. 2005, 52 (2), 303-306.

(24) Eiken Chemical Co. Ltd. Eiken Genome site, http://loopamp. eiken.co.jp/e/lamp/snps_index.html (accessed Jul 1, 2017).

(25) Zhang, C.; Yao, Y.; Zhu, J.-L.; Zhang, S.-N.; Zhang, S.-S.; Wei, H.; Hui, W.-L.; Cui, Y.-L. Sci. Rep. 2016, 6 (1), 26533-26539.

(26) Badolo, A.; Okado, K.; Guelbeogo, W. M.; Aonuma, H.; Bando, H.; Fukumoto, S.; Sagnon, N.; Kanuka, H. Malar. J. 2012, 11 (1), 227-233.

(27) Wang, D. Biotechnol. Biotechnol. Equip. 2016, 30 (2), 314-318.

(28) Duan, Y. B.; Yang, Y.; Wang, J. X.; Liu, C. C.; He, L. L.; Zhou, M. G. Sci. Rep. 2015, 5 (1), 17278-17288.

(29) Ayukawa, Y.; Hanyuda, S.; Fujita, N.; Komatsu, K.; Arie, T. Sci. Rep. 2017, 7 (1), 4253-4261.

(30) Lezhava, A.; Hayashizaki, Y. Single Nucleotide Polymorphisms. Methods in Molecular BiologyTM (Methods and Protocols); Humana Press: Totowa, NJ, 2009; pp 437-451.

(31) Talundzic, E.; Chenet, S. M.; Goldman, I. F.; Patel, D. S.; Nelson, J. A.; Plucinski, M. M.; Barnwell, J. W.; Udhayakumar, V. PLoS One 2015, 10 (8), 1-11.

(32) World Health Organization. World Malaria Report 2017; 2017.

(33) Aurrecoechea, C.; Brestelli, J.; Brunk, B. P.; Dommer, J.;

Fischer, S.; Gajria, B.; Gao, X.; Gingle, A.; Grant, G.; Harb, O. S.; et al. Nucleic Acids Res. 2009, 37 (SUPPL. 1), 539-543.

(34) Edgar, R. C. Nucleic Acids Res. 2004, 32 (5), 1792-1797.

(35) Kearse, M.; Moir, R.; Wilson, A.; Stones-Havas, S.; Cheung, M.; Sturrock, S.; Buxton, S.; Cooper, A.; Markowitz, S.; Duran, C.; et al. Bioinformatics 2012, 28 (12), 1647-1649.

(36) Nagamine, K.; Hase, T.; Notomi, T. Mol. Cell. Probes 2002, 16 (3), 223-229.

(37) Zadeh, J. N.; Steenberg, C. D.; Bois, J. S.; Wolfe, B. R.; Pierce, M. B.; Khan, A. R.; Dirks, R. M.; Pierce, N. A. J. Comput. Chem. 2011, 32 (1), 170-173.

(38) SantaLucia, J.; Hicks, D. Annu. Rev. Biophys. Biomol. Struct. 2004, 33 (1), 415-440.

(39) Bommarito, S.; Peyret, N.; SantaLucia, J. Nucleic Acids Res. 2000, 28 (9), 1929-1934.

(40) SantaLucia, J. Proc. Natl. Acad. Sci. U. S. A. 1998, 95 (4), 14601465.

(41) Wu, D. Y.; Ugozzoli, L.; Pal, B. K.; Qian, J.; Wallace, R. B. DNA Cell Biol. 1991, 10 (3), 233-238.

(42) Kwok, S.; Kellogg, D.; McKinney, N.; Spasic, D. Nucleic Acids Res. 1990, 18 (4), 999-1005.

(43) Yesilkaya, H.; Meacci, F.; Niemann, S.; Hillemann, D.; RuschGerdes, S.; Barer, M. R.; Andrew, P. W.; Oggioni, M. R. J. Clin. Microbiol. 2006, 44 (10), 3826-3829.

(44) Gaedigk, A.; Freeman, N.; Hartshorne, T.; Riffel, A. K.; Irwin, D.; Bishop, J. R.; Stein, M. A.; Newcorn, J. H.; Jaime, L. K. M.; Cherner, M.; et al. Sci. Rep. 2015, 5 (1), 9257-9265.

(45) Carlos, F. F.; Veigas, B.; Matias, A. S.; Doria, G.; Flores, O.; Baptista, P. V. Biotechnol. reports (Amsterdam, Netherlands) 2017, 16, 21-25.

(46) Badolo, A.; Bando, H.; Traoré, A.; Ko-ketsu, M.; Guelbeogo, W. M.; Kanuka, H.; Ranson, H.; Sagnon, N.; Fukumoto, S. Malar. J. 2015, 14 (1), 477-484.

(47) Itonaga, M.; Matsuzaki, I.; Warigaya, K.; Tamura, T.; Shimizu, Y.; Fujimoto, M.; Kojima, F.; Ichinose, M.; Murata, S. PLoS One 2016, 11 (3), e0151654.

(48) Chen, F.; Zhao, Y.; Fan, C.; Zhao, Y. Anal. Chem. 2015, 87 (17), 8718-8723.

(49) Imai, K.; Tarumoto, N.; Misawa, K.; Runtuwene, L. R.; Sakai, J.; Hayashida, K.; Eshita, Y.; Maeda, R.; Tuda, J.; Murakami, T.; et al. BMC Infect. Dis. 2017, 17 (1), 621-629.

(50) Han, E.-T.; Watanabe, R.; Sattabongkot, J.; Khuntirat, B.; Sirichaisinthop, J.; Iriko, H.; Jin, L.; Takeo, S.; Tsuboi, T. J. Clin. Microbiol. 2007, 45 (8), 2521-2528.

(51) Moser, N.; Rodriguez-Manzano, J.; Lande, T. S.; Georgiou, P. IEEE Trans. Biomed. Circuits Syst. 2018, 12, 1-12.

(52) Miscourides, N.; Yu, L.-S.; Rodriguez-Manzano, J.; Georgiou, P. IEEE Trans. Biomed. Circuits Syst. 2018, 1-13. 